\title{
DETERMINAÇÃO DE FUNÇÕES DE TRANSFERÊNCIA DE PROCESSOS QUÍMICOS ATRAVÉS DO MÉTODO DE EVOLUÇÃO DIFERENCIAL UTILIZANDO O SCILAB
}

\author{
A. H. R. REZENDE ${ }^{1}$, D. L. SOUZA ${ }^{1}$ \\ ${ }^{1}$ Universidade Federal do Triângulo Mineiro, Departamento de Engenharia Química \\ E-mail para contato: arthurhenriquerr@hotmail.com \\ RESUMO - Existem diversos métodos de otimização e as particularidades do \\ processo é que determina seu desempenho. Os métodos naturais de otimização \\ têm sido amplamente estudados. O objetivo deste trabalho é apresentar um estudo \\ do uso do método de otimização natural denominado Evolução Diferencial para a \\ obtenção de Funções de Transferência que descrevem o comportamento de um \\ processo.
}

\section{INTRODUÇÃO}

A modelagem consiste na determinação de um conjunto de equações matemáticas que possibilita a descrição do comportamento de um processo quando há a modifícação de parâmetros ou variáveis do mesmo. Uma forma de descrever o modelo de um sistema é empregando o conceito de Funções de Transferência, que será abordado posteriormente.

Otimização é um agrupamento de princípios e métodos matemáticos usados para resolver quantitativamente problemas em diversas disciplinas. O termo é utilizado para o aprimoramento de um certo processo, seja ele matemático ou experimental, a fim de extrair o rendimento mais satisfatório possível (OLIVEIRA, 2006).

O objetivo deste trabalho é estudar o método de otimização natural desenvolvido por Storn e Price (1995), conhecido como Evolução Diferencial (ED) bem como aplicar o método para obtenção de Funções de Transferência que representam o comportamento de um dado processo químico.

\section{Função de Transferência}

Função de Transferência (TF) é uma expressão algébrica para a relação dinâmica entre as entradas e as saídas de um modelo de processo. Ela é definida de forma independente das condições iniciais do sistema bem como de considerações particulares a respeito do modelo (SEBORG, 1989).

A Função de Transferência é obtida aplicando Transformada de Laplace na equação diferencial linearizada de determinado modelo. (SEBORG, 1989). 


\section{Evolução Diferencial}

O conceito principal desta técnica é o esquema proposto para atualização do vetor de variáveis de projeto de uma população. Essencialmente ocorre a diferença ponderada, por meio da taxa de perturbação $F$, entre dois indivíduos, $\overrightarrow{x_{r 2}}$ e $\overrightarrow{x_{r 3}}$, a qual é adicionada a um terceiro indivíduo, $\overrightarrow{x_{r 1}}$, como mostrado na Figura 1 (SOUZA et al, 2011).

Figura 1: Fundamentação teórica do algoritmo de ED (Fonte: Reproduzido de Storn et al., 2005).

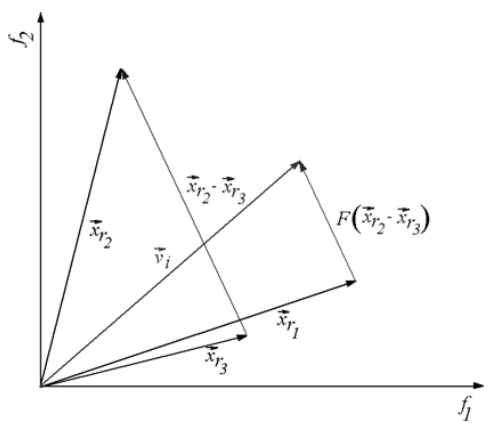

O indivíduo gerado através deste esquema $\left(\vec{v}_{i}\right)$ é avaliado pela função objetivo e pode inclusive substituir indivíduos mal sucedidos nas gerações seguintes. Desta forma, nenhuma distribuição de probabilidade em separado deve ser usada, o que torna este esquema completamente auto ajustável (SOUZA et al, 2011).

De maneira resumida, nessa abordagem puramente estrutural, o valor de cada variável é representado por um valor real e a geração de candidatos é feita através do uso de operações vetoriais como apresentado e detalhado a seguir (Storn e Price, 1995):

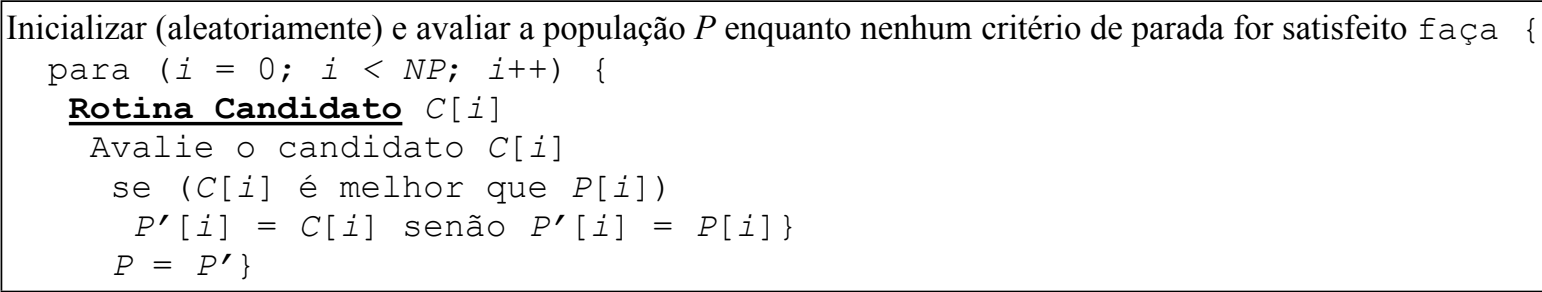

em que $N P$ é o tamanho da população, $P$ é a população da geração atual, e $P$ ' é a população da próxima geração. $O$ procedimento para a criação do candidato $(C[i])$ é mostrado a seguir:

\section{Candidato $\mathrm{C}[\mathrm{i}]$}

Aleatoriamente selecione os pais $P[i 1], P[i 2]$ e $P[i 3],(i \neq i 1 \neq i 2 \neq i 3)$.

Geração do candidato: $C 1[i]=P[i 1]+F \times(P[i 2]-P[i 3])$.

C[i] por cruzamento dos genes de $P[i]$ e $C l[i]$ como segue: para $(j=0 ; j<N P ; j++)\{$ se $(r<C R) C[i][j]=C 1[i][j]$ senão $C[i][j]=P 1[i][j]\}$ 
em que $C[i]$ é a solução candidata i da população, $C[i][j]$ é a $j$-ésima entrada no vetor solução de $C[i], r$ é um número aleatório entre 0 e $1, C R$ é a probabilidade de cruzamento e $F$ é a taxa de perturbação (SOUZA et al, 2011).

$\mathrm{Na}$ literatura pode-se encontrar diversas aplicações do algoritmo de ED, dentre quais pode-se citar: otimização de processos com controle ótimo (Souza et al, 2015), otimização de parâmetros da energia gerada em sistemas de biohidrogênio (HUANG et al, 2010), sintonia de controladores PID (SOUZA et al, 2011), determinação da difusividade térmica aparente na secagem das frutas (MARIANI et al, 2008), além de inúmeras aplicações em áreas distintas da ciência.

\section{Apresentação dos sistemas}

Para a realização do presente trabalho, utilizou-se de dois modelos matemáticos. O primeiro deles $\left(\mathrm{m}_{1}\right)$ é representado pela Figura 2 e simulado pela Equação 1.

Figura 2: Representação esquemática do modelo $\left(\mathrm{m}_{1}\right)$

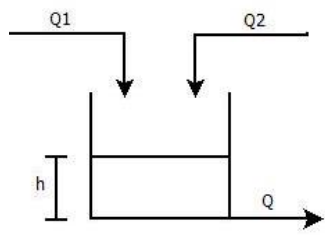

$\frac{d h}{d t}=\frac{Q 1}{A}+\frac{Q 2}{A}-\frac{\beta}{A} \sqrt{h}$

A Equação 2 representa a Função de Transferência do modelo $\mathrm{m}_{1}$.

$$
G(s)=\frac{6}{12 \cdot s+1}
$$

O segundo modelo utilizado no trabalho $\left(\mathrm{m}_{2}\right)$ é representado esquematicamente pela Figura 3 e simulado pelas Equações 3 e 4.

Figura 3: Representação esquemática do modelo (m2)

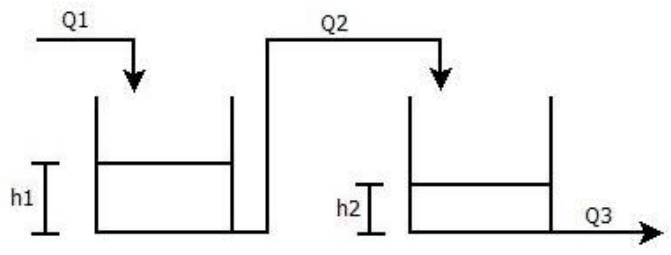

$$
\begin{aligned}
& \frac{d h_{1}}{d t}=\frac{Q_{1}}{A_{1}}-\frac{\beta_{1}}{A_{1}} h_{1} \\
& \frac{d h_{2}}{d t}=\frac{\beta_{1}}{A_{2}} h_{1}-\frac{\beta_{2}}{A_{2}} h_{2}
\end{aligned}
$$




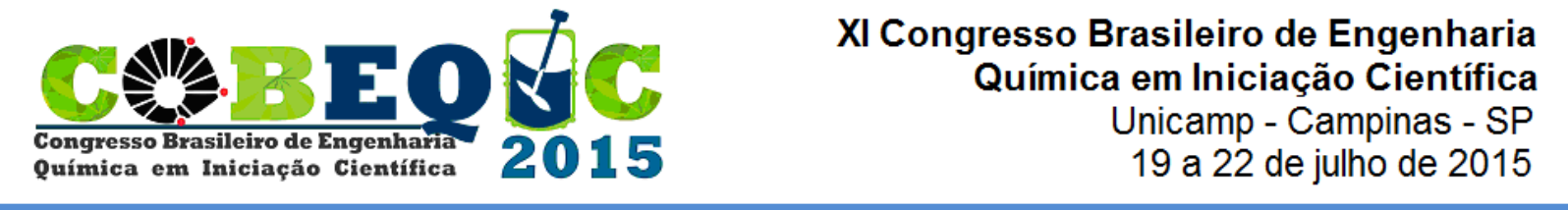

As Equações 5 e 6 descrevem as Funções de Transferência do modelo $\mathrm{m}_{2}$. $G 1(s)=\frac{0,3333}{0,666667 \cdot s+1}$

(5)

$$
G s(s)=\frac{1,5}{0,5 \cdot s+1}
$$

\section{RESULTADOS E DISCUSSÃO}

Em relação ao modelo $\mathrm{m}_{1}$, o algoritmo implementado em Scilab foi realizado com 500 iterações. A Tabela 1 apresenta os valores dos parâmetros da Função de Transferência obtidos na técnica de Evolução Diferencial e os valores teóricos da mesma equação algébrica. A Figura 4 representa visualmente os três diferentes comportamentos do modelo $\mathrm{m}_{1}$.

Tabela 1: Comparação dos valores dos parâmetros obtidos com os parâmetros teóricos $\left(\mathrm{m}_{1}\right)$

\begin{tabular}{ccccc}
\hline $\mathrm{m}_{1}$ & $\mathrm{ED}$ & $\mathrm{TF}$ & $\mathrm{R}^{2}$ & Erro Padrão \\
\hline $\mathrm{K}$ & 6,970 & 6,000 & 0,999 & 1,132 \\
$\tau$ & 14,598 & 12,000 & & \\
\hline
\end{tabular}

Figura 4: Representação Gráfica dos estudos do modelo $\left(\mathrm{m}_{1}\right)$

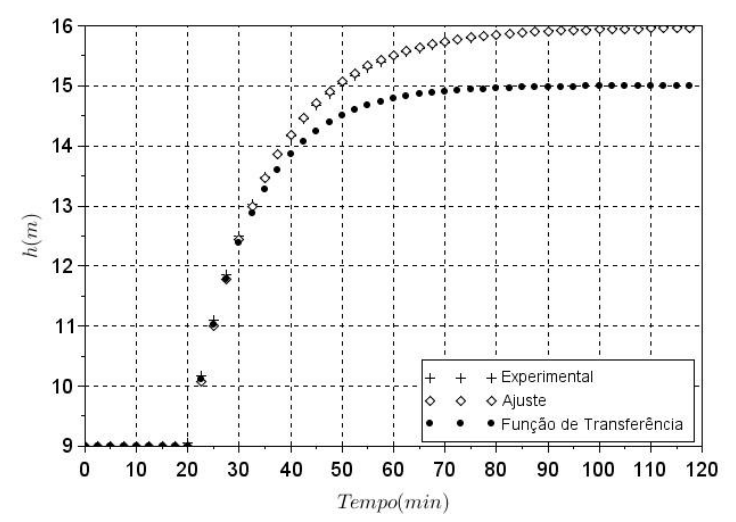

Com base na Tabela 1, percebe-se que a Função de Transferência obtida é expressa pela Equação 7.

$$
G(s)=\frac{6,970}{14,598 \cdot s+1}
$$

Observa-se a existência de uma diferença entre os valores dos parâmetros obtidos no ajuste e teóricos. Isso se deve ao fato de que para a obtenção da Função de Transferência pela Evolução Diferencial nenhuma operação matemática foi realizada diretamente no modelo. Com base na Figura 4 é possível perceber que a curva do ajuste mais se aproxima da curva do modelo $\mathrm{m}_{1}$, curva experimental, em comparação com a curva Função de Transferência. 
$\mathrm{O}$ modelo $\mathrm{m}_{2}$ foi implementado no Scilab nas mesmas configurações que o modelo $\mathrm{m}_{1}$. A Tabela 2 apresenta a comparação entre as Funções de Transferência obtidos no ajuste e os valores teóricos da mesma equação.

Tabela 2: Comparação dos valores dos parâmetros obtidos com os parâmetros teóricos (m2)

\begin{tabular}{cccc|c|c|c|c}
\hline $\begin{array}{c}\mathrm{m}_{2} \\
\text { tanque } 1\end{array}$ & $\mathrm{ED}$ & $\mathrm{TF}$ & $\mathrm{R}^{2}$ & $\begin{array}{c}\mathrm{m}_{2} \\
\text { tanque } 2\end{array}$ & $\mathrm{ED}$ & $\mathrm{TF}$ & $\mathrm{R}^{2}$ \\
\hline $\mathrm{K}$ & 0,332 & 0,333 & 0,998 & $\mathrm{~K}$ & 0,500 & 1,500 & \multirow{2}{*}{0,999} \\
$\tau$ & 0,5187 & 0,66667 & & $\tau$ & 1,000 & 0,500 & \\
\hline
\end{tabular}

Erro Padrão 0,0022

Figura 5: Representação Gráfica do tanque 1 do modelo $\mathrm{m}_{2}$

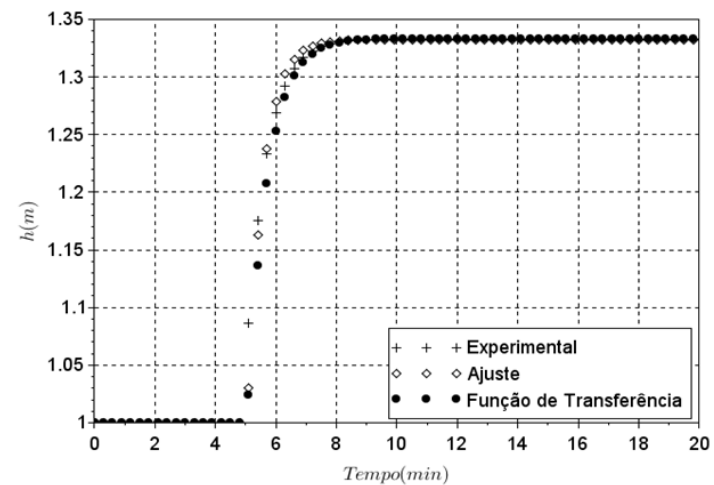

Figura 6: Representação Gráfica do tanque 1 do modelo m2

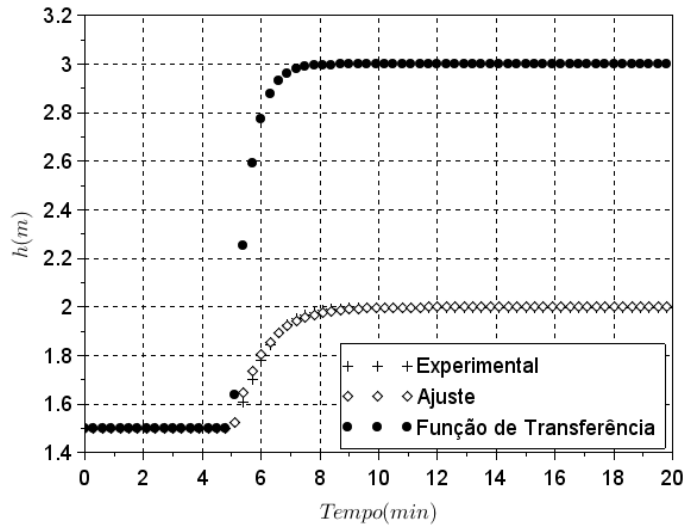

Com base na Tabela 2, percebe-se que as Funções de Transferência resultante para o modelo $\mathrm{m}_{2}$ é expressa pelas Equações 8 e 9 .

$$
G(s)=\frac{0,332}{0,5187 \cdot s+1}
$$




$$
G(s)=\frac{0,500}{1,00 \cdot s+1}
$$

Nota-se que existe uma diferença pouco satisfatória entre os valores dos parâmetros da Função de Transferência que descrevem o tanque 1 do modelo $m_{2}$. Com base na Figura 6 é possível perceber que as três curvas do gráfico praticamente se sobrepõe, o que mostra que os parâmetros das Funções de Transferência tanto do ajuste quanto do teórico podem representar o modelo experimental $\mathrm{m}_{2}$.

Em relação ao tanque 2 percebe-se um comportamento diferente do tanque 1, em que ocorre um desvio relevante nos parâmetros das Funções de Transferência. Percebe-se na Figura 7 que a curva da Função de Transferência teórica se diverge de forma considerável da curva experimental, enquanto a curva ajuste praticamente se sobrepõe a curva do modelo $\mathrm{m} 2$.

\section{CONCLUSÃO}

Neste trabalho foi possível aplicar o algoritmo de Evolução Diferencial para a obtenção de Funções de Transferência que representam o comportamento de um processo químico. A metodologia proposta foi aplicada ao estudo de nível de tanques em dois diferentes sistemas. Os parâmetros obtidos para o ajuste foram comparados com os parâmetros teóricos os quais apresentaram valores satisfatórios para os modelos. Além do mais, em relação a disposição gráfica dos modelos a curva do ajuste por ED se aproximou consideravelmente da curva experimental comprovando assim, que a aplicação desse algoritmo expressa resultados semelhantes ao modelo experimental.

\section{REFERÊNCIAS}

HUANG, S. R.; WU, C. C.; LIN, C. Y.; CHEN, H. T. Parameter Optimization of a Biohydrogen Real Time Power Generating System Using Differential Evolution Algorithm. Technical Communication, 2010.

MARIANI, V. C.; LIMA, A. G. B.; COELlO, L. S. C. Apparent Thermal Diffusivity Estimation of the Banana during Drying using Inverse Method. Journal of Food Engineering, 2008.

SOUZA, D. L.; MAIA, L. F. S.; LOBATO, F. S.; ALMEIDA, G. M. Sintonia de Controladores PID com o Algoritmo de Evolução Diferencial. Universidade Federal de São João del-Rei, 2011.

SOUZA, D. L.; LOBATO, F. S.; GEDRAITE, R. Robust Multiobjective Optimization Applied to Optimal Control Problems Using Differential Evolution. Chemical Engineering Technology, 2015.

SEBORG, D. E.; EDGAR, T. F.; MELliChAMP, D. A. Process Dynamics and Control. Wiley Series in Chemical Engineering, 1989.

STORN, R.; PRICE, K. Differential Evolution: A Simple and Efficient Adaptative Scheme for Global Optimization over Continuous Spaces. Technical Report TR-95-012, International Computer Science Institute, 1995. 
OLIVEIRA, G. T. S. Tese, Faculdade de Engenharia Mecânica, Universidade Federal de Uberlândia, Brasil 2006. 